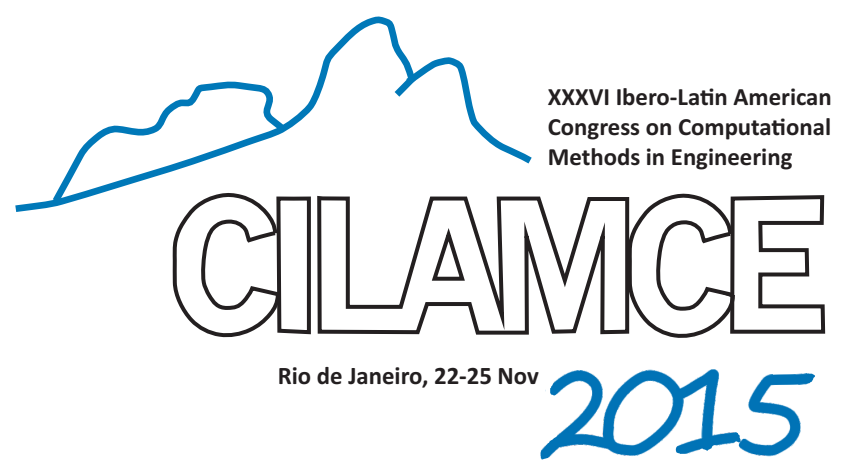

\title{
MULTI-SCALE SYSTEM RELIABILITY ANALYSIS
}

\section{Adelano Esposito}

\section{Herbert Martins Gomes}

adelano.esposito_@farroupilha.ifrs.edu.br

herbert@mecanica.ufrgs.br

Federal Institute of Education, Science and Technology of Rio Grande do Sul

Av. São Vicente, 95180-000, Farroupilha, Brazil

Mechanical Engineering Department, Federal University of Rio Grande do Sul;

Av. Sarmento Leite, 90050-170, Porto Alegre, Brazil

\begin{abstract}
The development of methods that allow to numerically reproduce the actual behavior of real structural systems has challenged scientists and researchers and urged them to address issues that extend beyond the integrity of a system. Therefore, a series of new parameters must be taken into account to evaluate the failure of a real structural system, especially in redundant systems. In the present paper, an accurate technique is developed to satisfy the probabilistic analysis. The proposed method estimates the reliability of the system with techniques that enable detecting multiple failure modes and, using the chi-squared distribution, a one direction exact integration is performed to calculate the failure probability of each mode in a more precise way than conventional approximation and simulation methods. This technique is based on the conventional (HDM) Hyperspace Division Method procedures which approximate the limit state surface by a series of spherical segments for provide accurate estimates of failure probabilities of components or systems. Besides, in this work, nonlinearity is considered by adopting an elastic plasticity model with linear hardening. Numerical applications showed the accuracy and efficiency of the methodology in the evaluation of the failure probability of problems represented by high nonlinear limit state functions with multiple failure regions.
\end{abstract}

Keywords: structural reliability, failure modes, redundant systems, nonlinearity, probabilistic analysis 


\section{INTRODUCTION}

The failure of redundant structural systems is usually preceded by a sequence of individual failures that describe several failure modes. To perform a system reliability analysis and to compute the system failure probability, it is necessary to use probabilistic methods capable of identifying dominant failure modes in the space of random variables. So far, many research efforts have been made to estimate the failure probabilities of structural systems, which depend on many factors, such as: (a) contribution of failure of one or more components to the overall system failure, (b) system redundancy, (c) behavior of the system after the failure of one or more components, (d) statistical correlation between events, and (e) progressive failure of components. Such system reliability analysis aims to compute the probability of the system failure event $E_{\text {sys }}$, which is often described as a cut-set system, i.e. (Kim et al., 2013):

$$
E_{\text {sys }}=\bigcup_{k=1}^{N_{\text {cut }}} C_{k}=\bigcup_{k=1}^{N_{\text {cut }}}\left[\bigcap_{i \in I_{C_{k}}} E_{i}\right]
$$

where $E_{i}$ is the $i$ th component event representing the failure at a location or member, $i=$ $1, \cdots, N_{\text {comp }} ; C_{k}$ is the $k$ th failure mode, $k=1, \cdots, N_{c u t}$ and $I_{c k}$ is the index set of components that appear in the failure mode $C_{k}$. In the practice, most structural systems are redundant, such that system failure has to be modeled by failure of a series of parallel subsystems (Imai et al., 2000).

Therefore, the approach developed in this paper based on the formulation in Eq. (1) can be used to compute the system failure probability for a series, parallel and hybrid (or seriesparallel) systems. Whose formulation considers the material nonlinearity due to the constitutive properties of materials, as well as the geometrical nonlinearity due to second order effects.

\section{STRUCTURAL RELIABILITY}

In the reliability analyses of a structural system, one investigates the probability of a structure not failing under a given load. In a limit state context, the failure of a structural element occurs when the effect of the load $(S)$ exceeds the resistance $(R)$. The assumption that $S$ exceeds $\mathrm{R}$ corresponds to the overlapping area between $f_{\mathrm{S}}(s)$ and $f_{\mathrm{R}}(r)$ (i.e. failure region), which represents the quantitative measure of failure probability ( see Fig. 1). Therefore, reliability can be given in terms of failure probability $\left(P_{f}\right)$ or reliability index $(\beta)$, as Eq. (2).

$$
P_{f}=\Phi(-\beta)=P(\mathrm{~S}>\mathrm{R})=P[g(\mathbf{X})<0]=\int_{g(\mathbf{X})<0} f(\mathbf{X}) \mathrm{d} \mathbf{X}=\int_{0}^{\infty} F_{\mathrm{R}(r)} f_{\mathrm{S}}(r) \mathrm{d} r
$$

where $\Phi$ is the standard normal distribution function, $\mathbf{X}$ represents the vector of stochastic variables of the reliability problem, $g(\mathbf{X})$ is the limit state function defining a safe state when $g(\mathbf{X})>0$ and a failure state when $g(\mathbf{X})<0$ - the hyper-surface separating the safe from the failure domain $g(\mathbf{X})=0$ is called the limit state,$- f_{\mathrm{S}}(r)$ is the probability density function of the load, and $F_{\mathrm{R}}(r)$ is the cumulative probability density function of the resistance. In general, the evaluation of the integral in Eq. (2) for an arbitrary failure region may not be possible. Therefore, approximation methods are obviously needed (Tran et al., 2014). In this study, 
the hyperspace division method (HDM) (Katsuki et al., 1994), is used to evaluate the failure probability. The key idea of the proposed method in the present paper and of the HDM is the same; however, the proposed method improves the previous and uses different tools to identify multiple points on the limit state surface. Once the limit state surface is detected, the method identifies the existing multiple failure modes, the range of failure probability for each failure mode and the overall failure probability of the system.

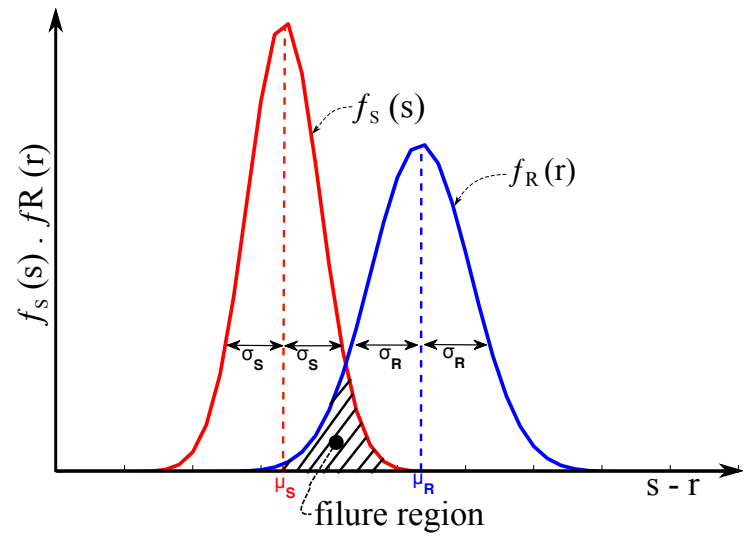

Figure 1. Distributions of load effects and structural resistance

\section{MULTIMODE FAILURE}

Structural systems can generally be characterized as series or parrallel systems or some combination of the two (hibrid system). In series system, the failure of any primary component causes the failure of the structure. The failure of the system is the union of the failure of primary components. For example, in statically determinate or rigid-plastic structures, formulation of a collapse mechanism will result in failure of the total system and therefore they can be modelled as series system with each element of the series being a failure mechanism. In parallel system, failure in a single element will not result in failure of the system, because the remaining elements may be able to sustain the external loads by redistributing of the loads. A typical example of a hibrid system is a statically indeterminate structure. Failure of such structures will always require that more than one element fails before the structure loses integrity and fails (Tran et al., 2014).

\section{SELECTIVE SEARCHING TECHNIQUE FOR DOMINANT FAIL- URE MODES}

For efficient system reliability evaluation, most of the existing failure-mode-based approaches employ approximation methods such as bounding formulas (Park et al., 2001; Ditlevsen et al., 1989), or response surface methods (Zhao et al., 1998). These methods may enable rapid estimation, but they are not flexible in incorporating various types and amount of available information on components or in accounting for statistical dependence among components and failure modes (Kim et al., 2013). According to (Kim et al., 2013), another challenge in such a system reliability analysis is that there often exist innumerable failure modes, i.e. possible combinations or sequences of components failures, because real structures are highly redundant and the failure of a member redefines the limit states of the remaining members (e.g. stress 
re-distribution caused by a member failure). Therefore, it is infeasible in most cases to enumerate all the possible failure modes for system reliability analysis (Kim et al., 2013). In order to overcome this difficulty, many studies have focused on the possibility of using only dominant failure modes with significant likelihood, instead of using all possible failure modes (Moses et al., 1979; Murotsu et al., 1984). Shao et al., 1999, proposed an improved simulation-based selective searching technique which genetic algorithm (GA) is used to find as small number of dominant failure modes. Kim et al., 2013, proposed a similar searching method to the one by (Murotsu et al., 1984), in that a GA scheme is used for search, but different in terms of two distinct GA search strategies such as searching direction and elitism. Therefore, dominant failure modes are obtained by a simulation-based selective searching technique employing a genetic algorithm the probabilities of the failure are computed by a matrix based system reliability (MSR) method. In Murotsu et al., 1984, is documented many methods developed to identify dominant failure modes.

\section{DIRECTIONAL SIMULATION}

Several methods for computing failure probabilities are available (Melchers, 1990), (Bjerager, 1990). In general, first and second order reliability methods (FORM and SORM) are extensively used (Hohenbichler et al., 1987). These methods are analytical and approximate probability integration approaches. They are based on replacing the limit state surface by firstand second-order surfaces fitted to the points at which the probability density has a local extremum (i.e, design points) in the transformed standard normal space (Katsuki et al., 1994). Note, however, that only when the limit state surface is sufficiently smooth in the standard normal space is it relatively easy and accurate to compute failure probabilities by FORM and SORM (Bjerager, 1990). Furthermore, when the probability density has multiple extremum points on the limit state surface the optimization algorithms used in FORM and SORM methods can not always guarantee the global maximum and, therefore, FORM and SORM do not necessarily yield the true solution to the approximate problem (Karamchandani et al., 1992). Also, as again specified by Bjerager, 1990, among others, FORM and SORM methods lose their accuracy for both highly nonlinear and system-reliability problems. Probably this imprecision is occasioned through the linearization of nonlinear limit state functions (Melchers et al., 2001). Katsuki et al., 1994, proposed a method that calculates the reliability of systems based on nonlinearities and multiple maximum points of the probability density on the limit state hypersurface. The Hyperspace Division Method (HDM) consists in approximating the actual limit state surface in the independent standard normal space by a series of hyperspherical segments, each having a radius described by a $\chi^{2}$ distribution. The failure probability was then approximated by the sum of the failure probability for both structural components and systems, the latter of which may have highly nonlinear limit states with multiple local extrema (Nie et al., 2000). The rationale for the proposed method in the present paper and for the HDM is the same; however, the proposed method utilizes different tools to identify the multiple points on the surface of the limit state function. Once the limit state surface is detected, the method identifies the existing multiple failure modes, the parcel of failure probabilities for each failure mode (whether an ultimate or serviceability failure mode) and the overall failure probability of the system (corresponding to the sum of $P_{f}$ of the ultimate failure modes). 


\section{FUNDAMENTAL PROCEDURE}

All directional methods require identification of directions along which the integration is performed in closed form, by simulation or numerical means. In the independent standard normal space, the integral along each direction is obtained exactly by using the $\chi^{2}$ distribution (Nie et al., 2000). The proposed method consists of five basic steps: (a) distribution points on the hypersphere; (b) search by intersection points $Q_{i}$ on the failure surface; (c) failure probability associated with the chi-square distribution; (d) contribution of each subdomain $P_{f i}$ to the total failure probability and (e) search technique for multimode failure.

\subsection{Even Distribution of Points on the Hypersphere}

For the purpose of distributing an arbitrary number of points uniformly on a hypersphere, six steps could be used: (a) the first step consists in generating $n$-points randomly; the points are generated on the surface of a hypersphere with a unit radius; (b) the respective coordinates are calculated for each point in the $n$-dimensional space; (c) the Euclidean distance of each point from its neighbors $\left(r_{m_{i}}\right)$ is then calculated; (d) the vector of repulsive force $\vec{f}$ of each point towards its neighbors is calculated. This repulsive force allows calculating the directional coordinates (with an increment of distance $s$, such that $s$ ranges from 0.0 to 1.0) of the resulting vector $\vec{f}_{n}$ indicating the update direction of point positions (i.e, $\vec{f}_{n}$ is interpreted as a move of point). After the move of point, the point coordinates are projected again onto the unit hypersphere coordinates. The process is iterative and is interrupted when the forces become balanced, i.e., when the repulsive force is not strong enough and therefore does not change the position of any of the points. Figure $2 b$ shows the methodological steps and a geometric representation in the three-dimensional space. The flowchart of the proposed searching technique is given in Fig. 2a, as explained in detail in this paragraph.

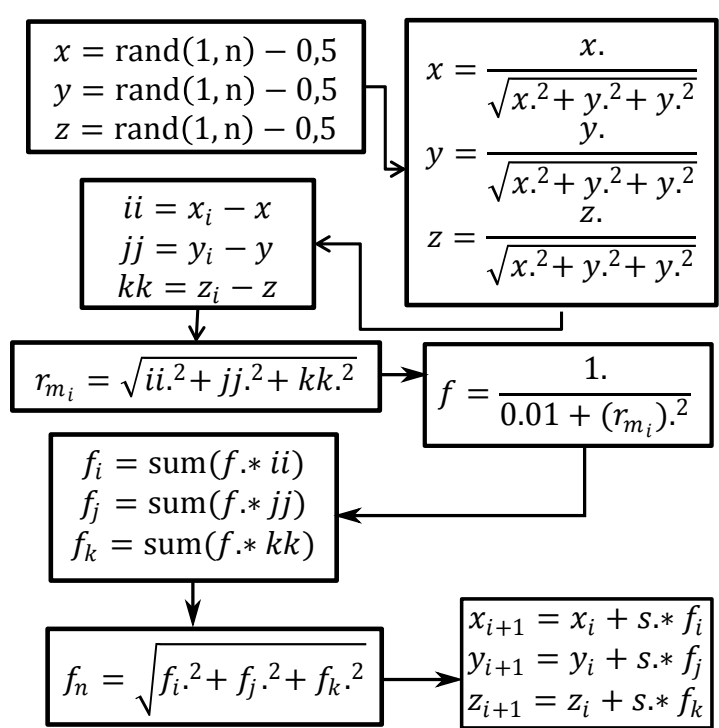

(a) Incremental-iterative procedure

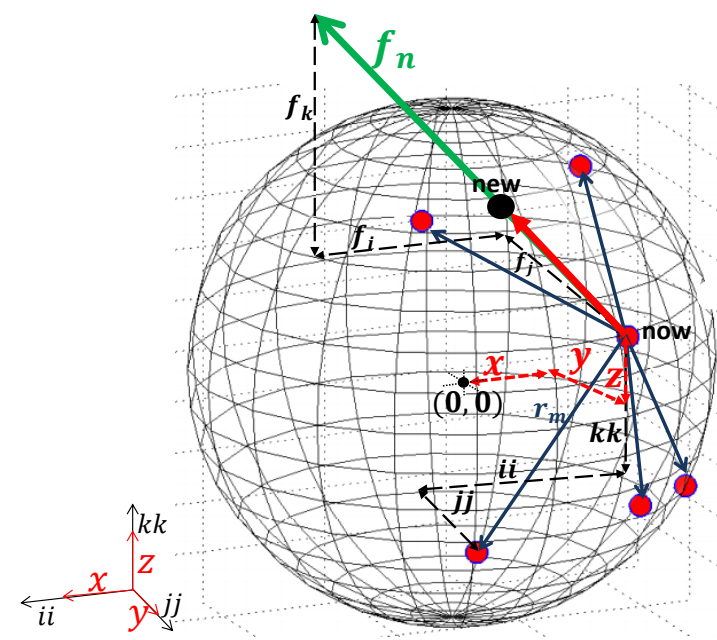

(b) Geometric solutions

Figure 2. Illustration of incremental-iterative procedure and distribution points 


\subsection{Search by Intersection Points $Q_{i}$ on the Failure Surface}

Polar coordinates of equidistant positions indicate the directions by which one conducts a one-dimensional search for the limit state function roots, which will be later used in the directional integration for each reliability analysis. The proposed method carries out this search through the bisection method. Since bisection permits finding a single root on a given interval, the technique was adapted so that it could be applied to the proposed method, thus allowing it to handle problems involving multiple roots or multiple limit state functions. The technique is summarized in the following steps:

a) Each unit direction vector $\mathbf{e}_{i}$ of length $\beta_{\max }$ is divided into identical $k$ subintervals such as $\Delta \beta=\beta_{\max } / k$;

b) The bisection method is performed as shown in Fig. 3a for each subinterval $\Delta \beta$. The lower and upper bounds of $\Delta \beta$ for a given direction $i$ are, respectively, $x_{i}^{j}$ and $x_{i}^{j+1}$, such that: $j=1,2, \cdots, k$;

c) After convergence of the bisection method to a given $\Delta \beta$ (i.e., $\left[x_{i}^{j}, x_{i}^{j+1}\right]$ ), the method is performed in the subsequent subinterval $\left(\left[x_{i}^{j+1}, x_{i}^{j+2}\right]\right)$ of the current direction $\mathbf{e}_{i}$. If the root is located, the polar coordinates of this position are kept, which correspond to point $Q_{i}$. The process is repeated until all subintervals have been assessed, i.e., $\left[x_{i}^{k-j}, x_{i}^{k}\right]$;

d) Once the method is applied along the whole length of direction $\mathbf{e}_{i}$, the following condition is checked: if any root is found, the process is repeated along the current direction $\mathbf{e}_{i}$; otherwise, the process takes place along the direction $\mathbf{e}_{i+1}$. In case of reset in the same direction $\mathbf{e}_{i}$, the limit state functions already intercepted along the current direction are deactivated, and hence, the method will search for the $\operatorname{root}(\mathrm{s})$ of another limit state function. It should be highlighted that only one limit state function is active during a given search in a given direction $\mathbf{e}_{i}$ (see Fig. 3b);

e) The process is repeated for a given direction $\mathbf{e}_{i}$ until all existing roots along this direction are found. After this condition is met, the method is carried out along the direction $\mathbf{e}_{i+1}$.

f) The search for the roots of the limit state function is made for all directions $\mathbf{e}_{i}$,such that: $i=1,2, \cdots, m$.

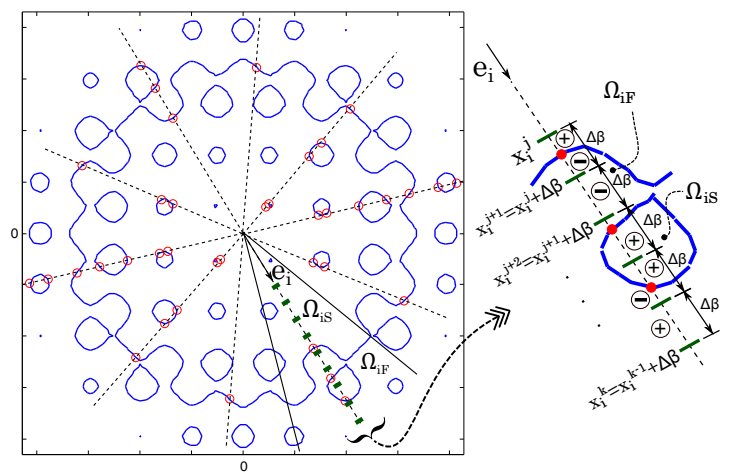

(a) Illustration of the failure surface and intersection points $Q_{i}$

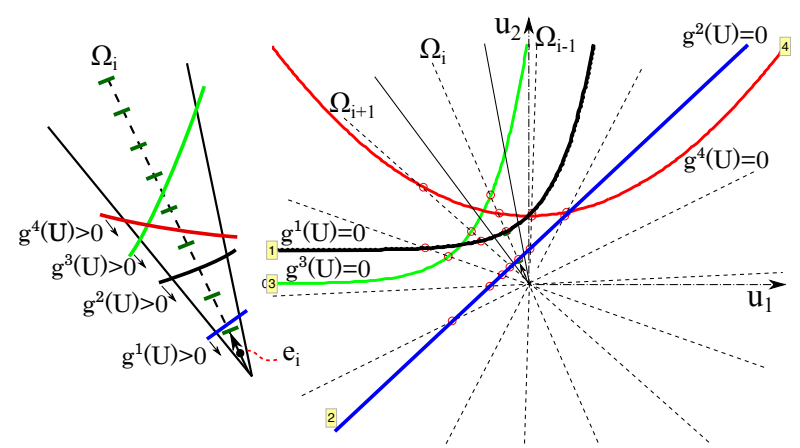

(b) Illustration of the multiple limit states and intersection points $Q_{i}$

Figure 3. Illustration of the intersection points $Q_{i}$ 


\subsection{Failure Probability Associated with the Chi-square Distribution}

After identifying the direction vectors $\mathbf{e}_{i}$, as well as the points of intersection between the centerline of a subdomain $\Omega_{i}$ and the limit state function $Q_{i}$ in the independent standard normal space, the integral along each direction is performed in closed form utilizing the $\chi^{2}$ distribution. If the limit state function is a hypersphere of radius $R$ in the hyperspace $\Omega$, then (Nie et al., 2000).

$$
g_{n}(\mathbf{U})=u_{1}^{2}-u_{2}^{2}-\cdots-u_{n}^{2}+R^{2}=-z^{2}+R^{2}=0
$$

and the failure probability associated with this hypersphere can be obtained exactly (Ang, 1984).

$$
P_{f}=P\left[g_{n}(\mathbf{U}<0)\right]=1-\chi_{n}^{2}\left(R^{2}\right)
$$

where $R=$ hypersphere radius and $\chi_{n}^{2}=$ cumulative distribution function of the chi-square distribution with $n$ degrees of freedom.

Figure $4 \mathrm{a}$, shows a two-dimensional standard normal space $\left(u_{1}, u_{2}\right)$ with a circular limit state function (the hypersphere limit state is associated with a two-dimensional space: $g_{n}=$ $-u_{1}^{2}-u_{2}^{2}+R^{2}=0$ ). The space is radially and equally split onto 16 subdomains that determine 16 equal arcs (hypersphere segments) on the limit state (Katsuki et al., 1994). If the hypersphere is radially divided evenly, the contribution of each subdomain $\left(P_{f i}\right)$ is the same. Therefore:

$$
P_{f i}=\left[1-\chi_{n}^{2}\left(R^{2}\right)\right] / m \quad \rightarrow \quad i=1,2, \cdots, m
$$

where $m$ is the total number of sections into which domain $\Omega$ will be split. In the more usual case where the limit state is a hypersurface rather than a hypersphere (Fig. 4), the hypersurface is approximated by a series of hyperspherical segments, each with its central point $Q_{i}$ located at the point of intersection between $\mathbf{e}_{i}$ with the current limit state function (Nie et al., 2000).

$$
P_{f i}=\left[1-\chi_{n}^{2}\left(R_{i}^{2}\right)\right] / m
$$

Considering that the failure probability of any subdomain $\Omega_{i F}$ is approximated by Eq. (6), the total failure probability $P_{f}$, which corresponds to the summation of all subdomains, and the reliability index $\beta$, are respectively:

$$
\begin{aligned}
& P_{f}=\sum_{i=1}^{m} P_{f i}=\sum_{i=1}^{m}\left[1-\chi_{n}^{2}\left(R_{i}^{2}\right)\right] / m \\
& \beta=\Phi^{-1}\left(1-P_{f}\right)
\end{aligned}
$$

where $R_{i}$ is the radius of the hypersphere segment of subdomain $i$.

\subsection{Contribution of Each Subdomain $P_{f i}$ to the Total Failure Probability}

The failure probability of a general system $P_{f_{s y s} g}$, concerns the calculation of the failure probability when the structure has more than one failure mode (multimode failure). Such problems arise when multiple performance functions $g^{k}, k=1,2, \cdots, k$, are imposed. This case 


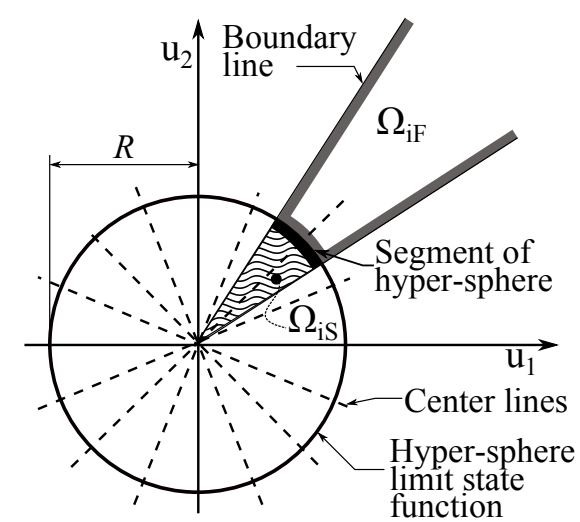

(a) Radial subdamains

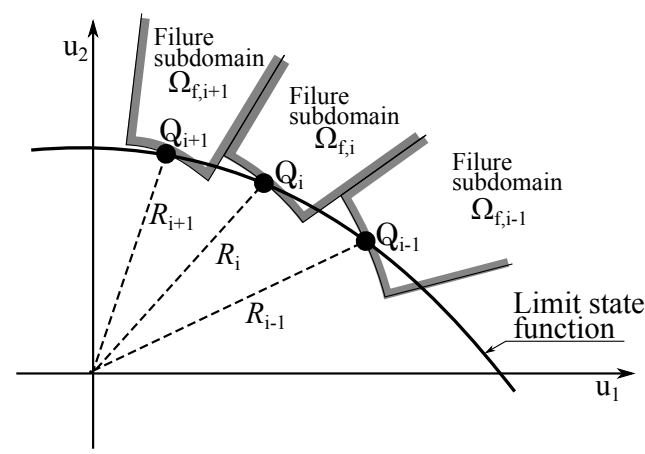

(b) Approximate nonlinear limit state function

Figure 4. Failure domain and segments for limit states circular and nonlinear: Adapted of Katsuki et al., 1994

is illustrated in Fig. 5 for four limit state functions in a two-dimensional standard normal space $\left(u_{1}, u_{2}\right)$. The failure probability of a general system is given by:

$$
P_{f_{s y s} g}=\sum_{i=1}^{m}\left[1-\chi_{n}^{2}\left(R_{\left(i, n_{f e l C}\right)}^{2}\right)\right] / m \quad \rightarrow \quad n_{f e l C}=1,2, \cdots, N_{f e l C}
$$

where $n_{f e l C}$ is the limit state function which corresponds to the critical element of the system and $N_{f e l C}$ is the total number of elements that cause the system to collapse.

As shown in Fig. 5, there exist two extreme cases of failure. The two extreme cases of series and parallel systems are associated with the minimum and maximum distances of the current limit state functions, i.e:

$$
\begin{aligned}
P_{f_{\text {sys } s}(i)} & =\left[1-\chi_{n}^{2}\left(\left(\min \left(R_{\left(i, n_{f e l}\right)}\right)\right)^{2}\right)\right] / m \\
P_{f_{s y s ~}(i)} & =\left[1-\chi_{n}^{2}\left(\left(\max \left(R_{\left(i, n_{f e l}\right)}\right)\right)^{2}\right)\right] / m
\end{aligned}
$$

where $P_{f_{s y s}(i)}$ and $P_{f_{s y s}(i)}$ are the total probabilities of failure associated with series and parallel systems, respectively, $R_{\left(i, n_{f e l}\right)}$ is the distance between the origin and the current limit state function $g^{n_{f e l}}$ along the $\mathbf{e}_{(.)}$, and $n_{f e l}$ is an $n$th limit state function.

In the proposed method, the evaluation of structural failure probability defined by Eq. (7) consists in splitting the domain in the standard normal space $\Omega$, into subdomain meshes $\Omega_{i}$, such that the volume of each $\Omega_{i, n_{f e l}}$ corresponds to the $P_{f}$ of the current limit state function $g^{n_{f e l}}$ along the centerline $\mathbf{e}_{i}$. Therefore, the contribution of each subdomain $\Omega_{i}$ to the total probability of failure by a limit state function in a two-dimensional standard normal space is search as follows:

- one calculates the volume below the joint probability density function (PDF), outside the limit state function (that consists in approximating of the current hypersurface by a hypersphere segment), between the bounds of the sector corresponding to the direction $\mathbf{e}_{i}$ (Fig. 5a). This procedure is carried out from the limit state function closest to the origin to the most distant one; 

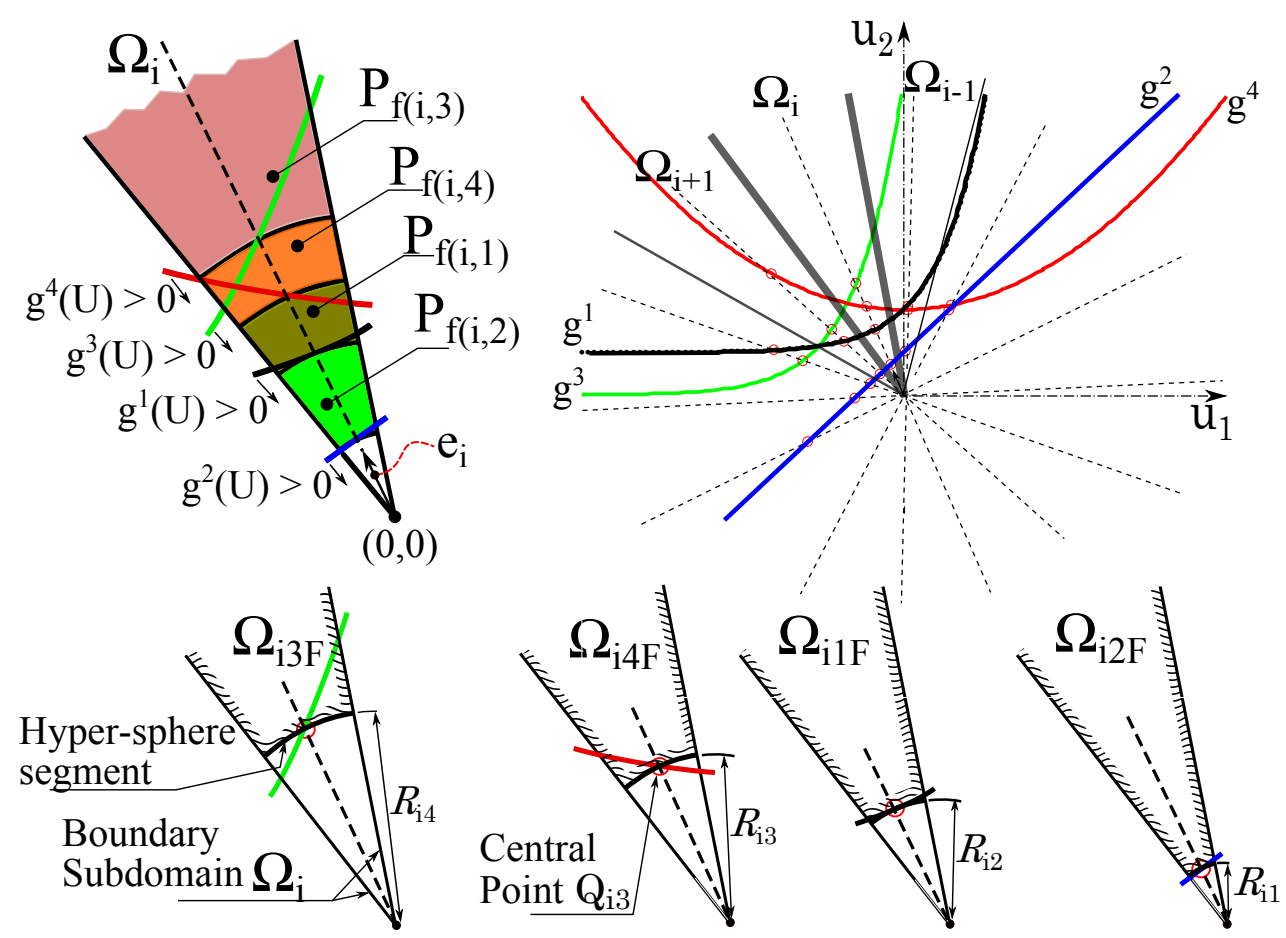

(a) Approximate multiple limit states by hyperspherical segments
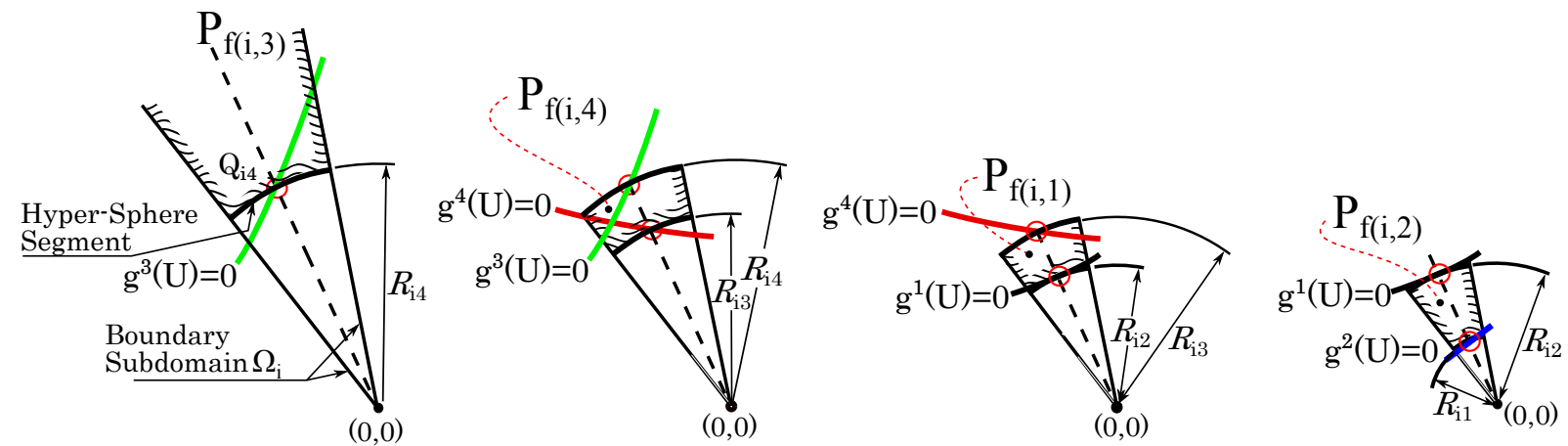

(b) Failure probability of any subdomain $\Omega_{i F}$

Figure 5. Multiple limit state functions and its approximations with spherical segments

- since the $P_{f}$ is determined solely by the distance from the origin $|\mathbf{U}|$, the limit state function closer to the origin is likely to contribute more to the system failure probability; therefore, the volume of the limit state function to the origin, along the direction $\mathbf{e}_{i}$, is subtracted from the volume of the second closest limit state function to the origin. This sequence is applied to all limit state functions along $\mathbf{e}_{i}$ (Fig. 5b).

Table 1 shows the formulation used to estimate the contribution of each subdomain mesh along the centerline $\mathbf{e}_{i}$ in Fig. 5.

\subsection{Search Technique for Multimode Failure}

The technique proposed in the previous section identifies the portions of $P_{f}$ in the decreasing order of their likelihood. Since the proposed searching method aims to capture multiple failure modes, the remaining tasks consist in performing a combination of failure sequences in order to identify dominant failure modes, also in the decreasing order of their likelihood. 
Table 1. Probability of failure to each subdomain mesh

\begin{tabular}{cc}
\hline Classification Systems & Formulation \\
\hline Parallel & $P_{f(i 3)}=\left[1-\chi_{n}^{2}\left(R_{i 4}^{2}\right)\right] / m$ \\
Series & $P_{f(i 2)}=\left[1-\chi_{n}^{2}\left(R_{i 1}^{2}\right)\right] / m$ \\
Hybrid or Parallel & $P_{f(i 3)}=\left[1-\chi_{n}^{2}\left(R_{i 4}^{2}\right)\right] / m$ \\
Hybrid & $P_{f(i 4)}=\left\{\left[1-\chi_{n}^{2}\left(R_{i 3}^{2}\right)\right] / m\right\}-\left\{\left[1-\chi_{n}^{2}\left(R_{i 4}^{2}\right)\right] / m\right\}$ \\
Hybrid & $P_{f(i 1)}=\left\{\left[1-\chi_{n}^{2}\left(R_{i 2}^{2}\right)\right] / m\right\}-\left\{\left[1-\chi_{n}^{2}\left(R_{i 3}^{2}\right)\right] / m\right\}$ \\
Hybrid & $P_{f(i 2)}=\left\{\left[1-\chi_{n}^{2}\left(R_{i 1}^{2}\right)\right] / m\right\}-\left\{\left[1-\chi_{n}^{2}\left(R_{i 2}^{2}\right)\right] / m\right\}$ \\
\hline
\end{tabular}

The failure modes causing the system failure are recorded and their occurrence frequencies are counted. Therefore, the orders of the failure modes correspond to the significance of the contribution to the system reliability of the truss structure. Table 2 shows the sequential failure modes obtained by the proposed method in the decreasing order of their occurrence.

Table 2. Failure modes of subdomain $\Omega_{i}$

\begin{tabular}{cc}
\hline Sequential Failure Modes & Probabilities of the Identified Failure Modes \\
\hline \multicolumn{1}{c}{2} & $P_{f(i 2)}=\left[1-\chi_{n}^{2}\left(R_{i 1}^{2}\right)\right] / m$ \\
$2 \rightarrow 1$ & $P_{f(i 2)}=\left\{\left[1-\chi_{n}^{2}\left(R_{i 1}^{2}\right)\right] / m\right\}-\left\{\left[1-\chi_{n}^{2}\left(R_{i 2}^{2}\right)\right] / m\right\}$ \\
$2 \rightarrow 1 \rightarrow 4$ & $P_{f(i 4)}=\left\{\left[1-\chi_{n}^{2}\left(R_{i 3}^{2}\right)\right] / m\right\}-\left\{\left[1-\chi_{n}^{2}\left(R_{i 4}^{2}\right)\right] / m\right\}$ \\
$2 \rightarrow 1 \rightarrow 4 \rightarrow 3$ & $P_{f(i 3)}=\left[1-\chi_{n}^{2}\left(R_{i 4}^{2}\right)\right] / m$ \\
\hline
\end{tabular}

\section{IMPLICIT LIMIT STATE FUNCTION AND SOURCES OF NON- LINEARITY}

If the structural response can be described analytically, the reliability can be evaluated In many cases of practical importance, particularly for complicated structures, the performance function $\mathrm{g}(\mathbf{X})$ is generally not available as an explicit, closed-form function of the input variables (Haldar, 2000). Therefore, the response has to be computed through a numerical procedure such as finite element analysis. The deterministic finite element method (FEM) consists of an iterative procedure to capture the nonlinear behavior of the structure (Haldar, 2000). The efficiency of the deterministic FEM is important for the success of the probabilistic method.

Most existing studies found in the literature, on the formulation of the numerical method incorporated into the probabilistic system, utilize the deterministic FEM model, assuming linear behavior for the structure. In this paper, the deterministic finite element method is developed considering sources of nonlinearity in a typical truss structure. Among the several sources of nonlinearity, the following stand out: (a) physical (or material) nonlinearity (Fig. 6a) and (b) geometrical nonlinearity (Fig. 6b). These nonlinearities will cause the configuration of a structure to deviate quite noticeably from its undeformed configuration. In such cases, linear 
analysis is obviously inappropriate, and nonlinear structural analysis should be used (Haldar, 2000).

In physical nonlinearity, the constitutive relation describing the material is itself nonlinear and the structural response associated with physical phenomena such as plasticity or strain softening must be captured. Physical nonlinearity is due to changes in geometry, arising from large strains and/or rotations, which enter the formulation from a nonlinear strain displacement relationship, and may occur even if the constitutive relation is linear (Leon et al., 2011).

Nonlinear problems arising from geometric or material nonlinearity feature critical points along the solution path. Critical points or stability points, shown in Fig. 6b, are points on the solution path where the structure loses stability or where bifurcation occurs. Load limit points occur when a local maximum or minimum load is reached on the load versus displacement curve, as shown at points $\mathrm{A}$ and $\mathrm{D}$ in Fig. 6b. A horizontal tangent is present at load limit points. Displacement limit points, shown at points B and C in Fig. 6b, occur at vertical tangents on the solution curve. Methods capable of run through displacement limit points are said to capture snap-back behavior. In this context, the unstable region following a load limit point corresponds to a physical instability in the structural system, such as buckling. Therefore, if snap-back behavior was not captured in Fig. 6b, the structure would appear to have a sharp drop in the load at point and the nature of the unloading might be lost (Leon et al., 2011).

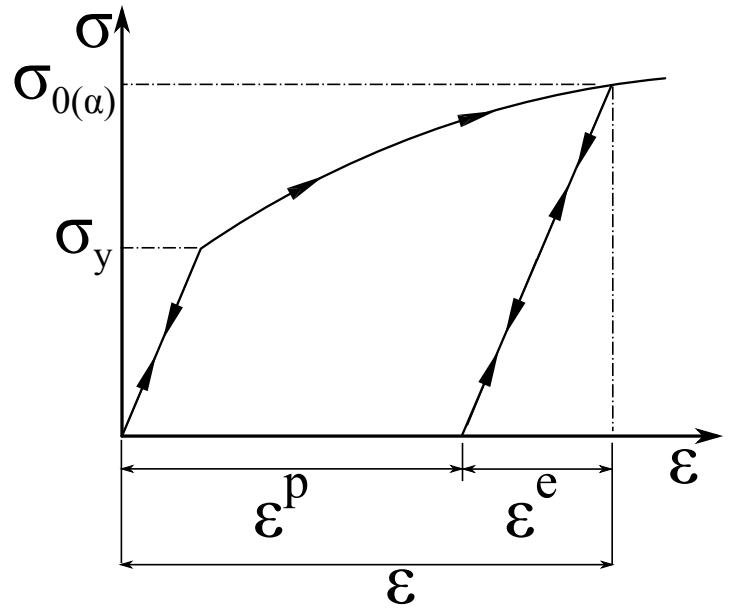

(a) Material nonlinearity: elasto-plasticity

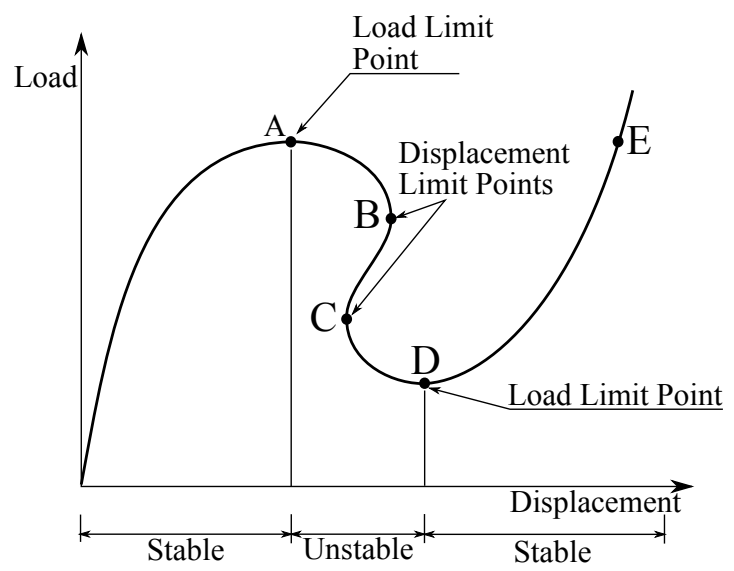

(b) Critical points in nonlinear equilibrium paths

Figure 6. Material and geometrical nonlinearity

Usually, the solution to non-linear problems is obtained by the use of incremental and iterative techniques, which should be able to follow the equilibrium path beyond critical points; therefore, most of the methods will trace the equilibrium path point by point, through an iterative scenario. Most of these techniques are a natural extension of the well-known Newton-Raphson iterative method, such as: load control, displacement control, arc-length control, and generalized displacement control method (GDCM). The GDCM was initially proposed, Yang et al., 1990, as an alternative to the existing methods for solving non-linear problems with multiple limit and snap-back points (Cardoso et al., 2006). In this work, we use the GDCM to solve a complex non-linear problem. For further discussion on non-linear systems, the reader is referred to Leon et al., 2011; Yang et al., 1994; Gu, 2004; Chen, 2008 and Bathe, 1996. 


\section{NUMERICAL EXAMPLES}

To verify the accuracy of propose method, two examples were analysed: one explicit function and one statically undertermined 6-bar truss. The efficiency and accuracy of the proposed method are compared with the Monte Carlo simulation and with the best results in literature.

Problem 1 is the same example used by Sorensen, 2004, which consist of a parallel system of four failure elements. After the transformation of the stochastic variables $x_{1}$ and $x_{2}$ into the standard normal space of variables $u_{1}$ and $u_{2}$, the four failure elements are described by the following failure functions: $g_{1}(\mathbf{U})=\exp \left(\mathrm{u}_{1}\right)-\mathrm{u}_{2}+1 ; g_{2}(\mathbf{U})=\mathrm{u}_{1}-\mathrm{u}_{2}+1 ; g_{3}(\mathbf{U})=$ $\exp \left(\mathrm{u}_{1}+2\right)-2 ; g_{4}(\mathbf{U})=0.1 \mathrm{u}_{1}^{2}-\mathrm{u}_{2}+2$. The failure functions, are shown in Fig. 7.
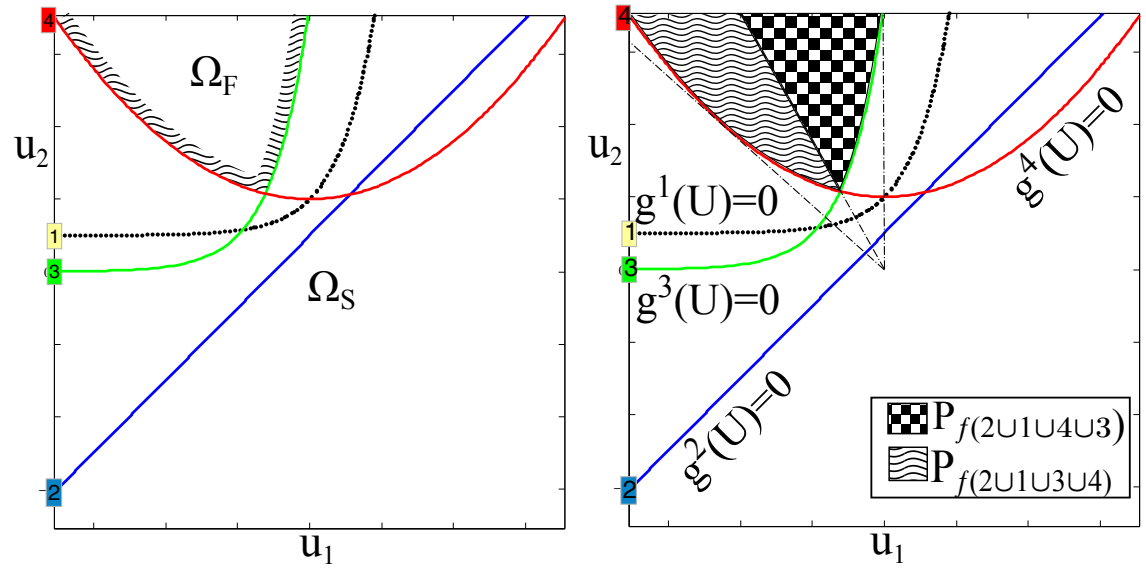

Figure 7. Failure domain for a parallel system

For illustration purposes, Fig. 7 shows the domain of a failure mode in the two-dimensional standard normal space. The lines show the limit state surfaces of component events while the space enclosed by limit state surfaces represent the failure mode. Table 3 shows the sequences of components failures (column 1) and reliability index (column 2) of the proposed method. The "exact" solution $\beta=3.56399$ (by chi-square distribution) or $\beta=2.92092$ (by normal distribution) was obtained by the Monte Carlo simulation using one million simulation runs, when the coefficient of variation (c.o.v) reaches 0.02 . The reliability index calculated by Sorensen, 2004 , is 2.92 . Note that the proposed procedure converges very quickly to a probability estimate close to that obtained by the Monte Carlo analysis and Sorensen, 2004.

Problem 2 is a practical engineering example (see Fig. 8). It consists of six-member truss structure as shown in Fig. 8a. The data on loads and materials are listed in Tab. 4. All the random variables are assumed to follow the independent normal distribution except for load L5, which is assumed to follow a lognormal distribution. The material and geometrical properties are elastic modulus $E=210 \mathrm{GPa}$, isotropic hardening parameter (plastic modulus) $H=210 \mathrm{MPa}$, cross sectional area of each truss member $2.3 \times 10^{-4} \mathrm{~m}^{2}$. The constitutive laws adopted for materials are shown in Fig. 8b. The stress-strain diagram of steel is assumed elastic plastic with bilinear hardening both in tension and in compression.

The truss is a statically indeterminate structure in which the failure of any two members results in structural collapse. Kim et al., 2013 and Shao et al., 1999, using the same example, in which all the elements are assumed to have ideal elasto-plastic behavior. However, in this work, is considered an elastic plasticity model with linear hardening. The failure modes identified by 
Table 3. Reliability index and failure probability of failure modes and overall system

\begin{tabular}{ccc}
\hline \multirow{2}{*}{ Ultimate Failure Modes } & \multicolumn{2}{c}{ Proposed Method } \\
\cline { 2 - 3 } $2 \rightarrow 1 \rightarrow 4 \rightarrow 3$ & 3.7519 & Failure Probabilities \\
\hline $2 \rightarrow 1 \rightarrow 3 \rightarrow 4$ & 3.7704 & $8.1843 \times 10^{-4}$ \\
System & 3.5720 & $1.6959 \times 10^{-3}$ \\
\hline Serviceability Failure Modes & & \\
\hline 2 & 1.8117 & $1.9376 \times 10^{-1}$ \\
$2 \rightarrow 4$ & 3.2057 & $5.8681 \times 10^{-3}$ \\
$2 \rightarrow 4 \rightarrow 1$ & 3.4444 & $2.6537 \times 10^{-3}$ \\
$2 \rightarrow 3$ & 3.4638 & $2.4811 \times 10^{-3}$ \\
$2 \rightarrow 1$ & 2.8255 & $1.8468 \times 10^{-2}$ \\
$2 \rightarrow 1 \rightarrow 3$ & 3.2219 & $5.5701 \times 10^{-3}$ \\
$2 \rightarrow 3 \rightarrow 1$ & 3.5996 & $1.5362 \times 10^{-3}$ \\
$2 \rightarrow 1 \rightarrow 4$ & 3.1294 & $7.4728 \times 10^{-3}$ \\
4 & 3.8146 & $6.9231 \times 10^{-4}$ \\
$4 \rightarrow 2$ & 4.0978 & $2.2576 \times 10^{-4}$ \\
\hline
\end{tabular}

the proposed method, together with the value obtained for the reliability index, are presented in Tab. 5.

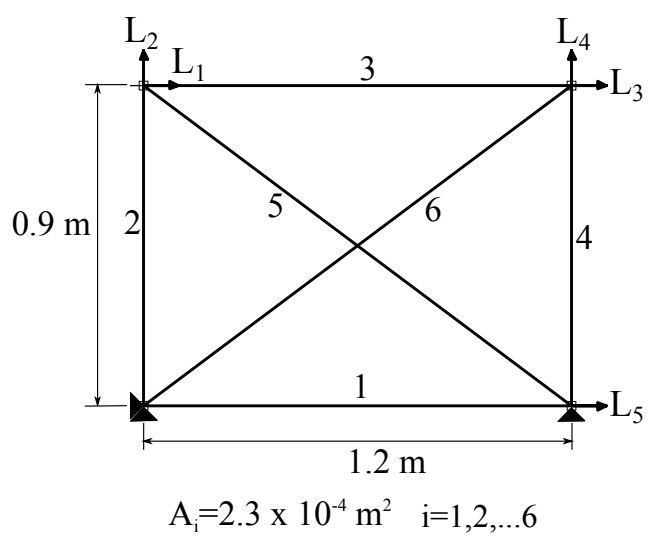

(a) Six-bar truss

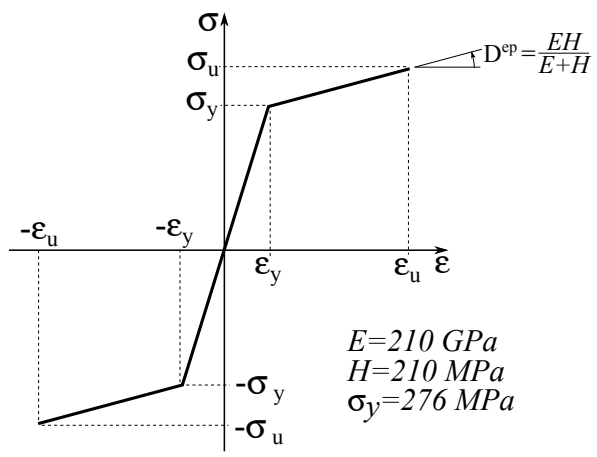

(b) Stress-strain diagrams of the material

Figure 8. Six-bar truss and constitutive laws adopted for materials

Since the structure collapses only after the failure of a second member, the sequences of failures that make up the ultimate failure mode are expected to involve at least two members. This can be confirmed in the first column of Tab. 5, which displays the respective sequences of failures that cause the structure to collapse. The second column shows the respective relia- 
Table 4. Statistical parameters and distribution types

\begin{tabular}{cccc}
\hline Random Variables & Distribution & Mean & c.o.v \\
\hline$L_{1}$ & Normal & $50 \mathrm{kN}$ & 0.1 \\
$L_{2}$ & Normal & $30 \mathrm{kN}$ & 0.1 \\
$L_{3}$ & Normal & $20 \mathrm{kN}$ & 0.1 \\
$L_{4}$ & Normal & $30 \mathrm{kN}$ & 0.1 \\
$L_{5}$ & Lognormal & $20 \mathrm{kN}$ & 0.1 \\
$\sigma_{y i}$ & Normal & $276 \mathrm{MPa}$ & 0.05 \\
\hline
\end{tabular}

Table 5. Reliability index and number for simulations of failure modes and overall failure of the system

\begin{tabular}{ccccc}
\hline Proposed Method & \multicolumn{3}{c}{ Kim et al., 2013 } & \multicolumn{2}{c}{ MCS } \\
\hline Ultimate Failure Modes & $\beta$ & Ultimate Failure Modes & $\beta$ & $\beta$ \\
\hline $6 \rightarrow 2$ & 3.1142 & $6 \rightarrow 2$ & 3.0735 & 3.0700 \\
$2 \rightarrow 6$ & 4.5334 & $2 \rightarrow 6$ & 3.4300 & - \\
$6 \rightarrow 1$ & 3.5110 & $6 \rightarrow 1$ & 3.4346 & 3.4980 \\
$6 \rightarrow 5$ & 6.3732 & - & - & - \\
System & 3.0189 & & 2.9775 & 3.0330 \\
\hline Serviceability Failure Modes & & & & \\
\hline 6 & 1.2902 & - & - & \\
2 & 3.5533 & - & - & \\
1 & 5.2863 & 28400 & & $1.0 \times 10^{6}$ \\
\hline
\end{tabular}

bility indices $(\beta)$ for each ultimate failure sequence. Based on this information, it is possible to note that the largest incidence of failures is associated with member 6 (initially) and, after the redistribution of efforts, with member 2. The least probable sequence of failures that could cause the structure to collapse is given by failure in member 6 followed by failure in member 5. To estimate the overall failure probability of the system, adding the parcels of probabilities corresponding to each ultimate failure sequence would suffice. The product of this sum corresponds to the overall failure probability $\left(P_{f_{s y s} g}\right)$, which is represented in 5 in terms of reliability index $\beta=3.0189$. These results may be compared with those obtained by Kim et al., 2013, indicating similarities between the sequences in the ultimate failure mode and the proximity between the reliability indices for each sequence and for the ultimate failure of the system, i.e. $\beta_{\text {Proposed Method }}=3.0189 \times \beta_{\text {Benchmark }}=2.9775$. As the Monte Carlo simulation (MCS) is the method that best solves the problem, it was used to obtain the overall reliability index of the 
system $\beta_{\text {sys } g}=3.0330$, which is fairly close to that obtained by the proposed method.

The serviceability failure modes are also shown in Tab. 5. A serviceability failure consists of a single element. The results described in the respective table confirm that the highest incidence of serviceability failure is that which occurs in member 6 , corresponding to a reliability index equal to $\beta=1.2902$.

Finally, it is possible to assess the proposed method as to the number of simulations necessary to perform the probabilistic analysis of the problem. Its numerical efficiency is investigated to examine the number of simulations required to identify the ultimate failure mode (structural collapse). Note in Tab. 5 that 2983 callbacks to the structural analysis program were necessary in the proposed method, compared with 28400 in the method developed by Kim et al., 2013 and $1.0 \times 10^{6}$ with the MCS. These results confirm that the proposed method greatly improves the computational efficiency both in searching failure modes and in evaluating the system failure probability.

\section{CONCLUSIONS}

This paper introduced an iterative approach: (a) to identify the overall failure modes in the system, which lead the structure to a catastrophic failure, (b) to identify serviceability failure modes, and (c) to identify the parcels of failure probability for the respective failure modes. This technique is based on the HDM, which consists in splitting the stochastic domain into an array of sectors, with approximation of the limit state function by a spherical segment for each sector. The accuracy of the HDM depends directly on the equidistance between the sectors. Hence, this paper presented a procedure to generate these "evenly distributed" distances (or directions). Numerical applications showed the accuracy and performance of the methodology. The proposed method proved accurate in solving the problem involving explicit limit state functions compared with the MCS and with the results of the referenced author. In this case, it was possible to carry out a graphical analysis of the failure regions, corroborating that the sequence found by the method for the failure modes is the same as that shown in the graphical representation of the problem. Finally, the method was submitted to the solution of a probabilistic problem involving a statically indeterminate 6-member structure with some degree of redundancy. In this case, the results demonstrated that the method detected the failure modes that match those obtained by the referenced author, as well as similar values for the overall reliability index. The MCS was also implemented to confirm the accuracy of the reliability index. This analysis allows checking the accuracy of the method proposed for the solution of problems involving multiple explicit limit state functions as well as of actual redundant problems, necessarily taking into account their physical nonlinearities in the structural analysis. Finally, the superior performance of the proposed method is demonstrated in comparison with the example provided by Kim et al., 2013, and independent evaluations by Monte Carlo simulation.

\subsection{Permission}

The authors are the only responsible for the printed material included in this paper.

\section{REFERENCES}

Ang, A. H-S., 1984. Probability Concepts in Engineering Planning and Design. vol. 2. John Wiley and Sons Inc. 
Bathe, K-J., 1996. Finite Element Procedures. 2 nd ed. Prentice-Hall International Series in. Prentice Hall.

Bjerager, P., 1990. On Computation Methods for Structural Reliability Analysis. Structural Safety, vol. 9, pp. 79-96.

Cardoso, E. L. and Fonseca, J. S. O., 2006. The gdc method as an orthogonal arc-length method. Communications in Numerical Methods in Engineering, vol. 23, no. 4, pp. 263-271.

Chen, W. F., 2008. Structural engineering: Seeing the bing picture. KSE Journal of Civil Engineering, vol. 12, no. 1, pp. 25-29.

Ditlevsen, O., 1979. Narrow reliability bounds for structural system. Journal of Structural Mechanics, vol. 7, no. 4, pp. 453-72.

Feng, Y., 1989. A method for computing structural system reliability with high accuracy. Computers and Structures, vol. 33, no. 1, pp. 1-5.

Gu, J., 2004. Large Displacement Elastic Analysis of Space Frame Allowing for FlexuralTorsional Buckling of Beans. PhD. Dissertation, Hong Kong Polytechnic University.

Haldar, A., 2000. Reliability Assessment Using Stochastic Finite Element Analysis. New York: John Wiley.

Hohenbichler, M., Gollwitzer, S., Kruse, W. and Rackwitz, R., 1987. New Light on First-And Second-Order Reliability Methods. Structural Safety, vol. 4, pp. 267-284.

Imai, K. and Frangopol, D. M., 2000. Geometrically nonliear finite element reliability analysis of structural systems. I: theory. Computer and Structures, vol. 1, no. 77, pp. 677-691.

Karamchandani, A. and Cornell, C. A., 1992. Sensitivity estimation within first and second order reliability methods. Structural Safety, vol. 11, no. 2, pp. 95-107.

Katsuki, S. amd Frangopol, D. M., 1994. Hyperspace Division Method for Structural Reliability. Journal of Engineering Mechanics, vol. 120, no. 11, pp. 2405-2427.

Kim, D-S, Ok, S-Y, Song, J. and Koh, H-M., 2013. System Reliability Analysis Using Dominant Failure Modes Identified by Selective Searching Technique. Reliability Engineering and System Safety, pp. 316-331.

Leon, S., E., Paulino, G. H., Pereira, A., Menezes, I. F. M. and Lages, E. N., 2011. A Unified Library of Nonlinear Solution Schemes. ASME, vol. 64, no. 4, pp. 26.

Melchers, R., 1990. Radial Importance Sampling for Structural Reliability. Journal of Engineering Mechanics, vol. 116, no. 1, pp. 189-203.

Melchers, R. and Ahammed, M., 2001. Estimation of Failure Probabilities for Intersections of Non-linear Limit States. Structural Safety, vol. 23, no. 2, pp. 123-135.

Moses, F. and Stahl, B., 1979. Reliability Analysis Format for Offshore Structures. Journal of Petroleum Technology, vol. 31, no. 3, pp. 347-354.

Murotsu, Y., Okada, H., Taguchi, K., Grimmelt, M. and Yonezawa, M., 1984. Automatic generation of stochastically dominant failure modes of frame structures. Structural Safety, vol. 2, no. 1 , pp. 17-25.

Nie, J. and Ellingwood, B.R., 2000. Directional methods for structural reliability analysis. 
Structural Safety, pp. 233-249.

Park, S. Y., 2001. A New Methodology for the Rapid Calculation of System Reliability of Complex Structures. Architectural Research, vol. 3, no. 1, pp. 71-80.

Shao, S. and Murotsu, Y., 1999. Approach to Failure Mode Analysis of Large Structures. Probabilistic Engineering Mechanics, pp. 169-177.

Sorensen, J. D., 2004. Structural Reliability Theory And Risk Analysis.

Tran, T. N. and Staat, M., 2014. Uncertain multimode failure and limit analysis of shells. 11th World Congress on Computational Mechanics (WCCM XI), 5th European Conference on Computational Mechanics (ECCM V), 6th European Conference on Computational Fluid Dynamics (ECFD VI).

Yang, Y-B. and Kuo, S-R., 1994. Theory and Analysis of Nonlinear Frame Structures. 1 nd ed. Prentice Hall.

Yang, Y-B. and Shieh, M-S., 1990. Solution Method for Nonlinear Problems with Multiple Critical Points. AIAA Journal, vol. 28, no. 12, pp. 2110-2116.

Zhao, Y. G. and Ono, T., 1998. System reliability evaluation of ductile frame structures. Journal of Structural Engineering, vol. 124, no. 6, pp.678-85. 